\title{
LARGE TIME BEHAVIOUR OF SOLUTIONS TO NONHOMOGENEOUS DIFFUSION EQUATIONS
}

\author{
JEAN DOLBEAULT \\ Ceremade (UMR CNRS no. 7534), Université Paris Dauphine \\ Place de Lattre de Tassigny, 75775 Paris Cédex 16, France \\ E-mail:dolbeaul@ceremade.dauphine.fr \\ http://www.ceremade.dauphine.fr/ dolbeaul \\ GRZEGORZ KARCH \\ Instytut Matematyczny, Uniwersytet Wrockawski \\ Pl. Grunwaldzki 2/4, 50-384 Wrockaw, Poland \\ E-mail: karch@math.uni.wroc.pl \\ http://www.math.uni.wroc.pl/ karch
}

\begin{abstract}
This note is devoted to the study of the long time behaviour of solutions to the heat and the porous medium equations in the presence of an external source term, using entropy methods and self-similar variables. Intermediate asymptotics and convergence results are shown using interpolation inequalities, Gagliardo-Nirenberg-Sobolev inequalities and Csiszár-Kullback type estimates.
\end{abstract}

1. Introduction. In this note, we study the large time behavior in $L^{1}\left(\mathbb{R}^{N}\right)$ of solutions to the Cauchy problem for the porous media equation $(m>1)$ and the heat equation $(m=1)$ in the presence of an external source term:

$$
\begin{aligned}
& v_{t}=\Delta v^{m}+G(x, t) \quad x \in \mathbb{R}^{N}, \quad t>0, \\
& v(x, 0)=v_{0}(x) .
\end{aligned}
$$

2000 Mathematics Subject Classification: Primary 35K05, 35K65; Secondary 35B40, 35E05, 94A17, 76S05, 76R50.

Key words and phrases: large time behaviour, nonhomogeneous equations, heat equation, porous media equation, nonlinear diffusions, $L^{1}$ estimates, self-similar solutions, Barenblatt solutions, intermediate asymptotics, self-similar variables, stationary solutions, Fokker-Planck type equations, relative entropy methods, Gagliardo-Nirenberg inequalities, logarithmic Sobolev inequality, Gagliardo-Nirenberg-Sobolev inequality, Csiszár-Kullback inequality.

The paper is in final form and no version of it will be published elsewhere. 
Here, we always assume that $v_{0} \in L^{1}\left(\mathbb{R}^{N}\right)$ and $G \in L^{1}\left(\mathbb{R}^{N} \times[0, T]\right)$ for every $T>0$. For $m=1$, the solution of problem (1)-(2) is given by the well-known Duhamel formula. On the other hand, in the nonlinear case $m>1$ the unique solution to (1)-(2) can be obtained e.g. via the theory of nonlinear semigroups, cf. [20].

Concerning the large time behavior of solutions, it is known that under the additional assumption $G \in L^{1}\left(\mathbb{R}^{N} \times[0, \infty)\right)$, we have

$$
\lim _{t \rightarrow \infty}\left\|v(\cdot, t)-E_{M_{\infty}}(\cdot, t)\right\|_{1}=0
$$

where the $L^{1}$-norm is denoted by $\|\cdot\|_{1}$ and $E_{M_{\infty}}$ is the source-type (or fundamental) solution to the homogeneous problem

$$
E_{t}=\Delta E^{m}, \quad E(0)=M_{\infty} \delta_{0}
$$

with mass

$$
M_{\infty}:=\lim _{t \rightarrow \infty} \int_{\mathbb{R}^{N}} u(x, t) d x=\int_{\mathbb{R}^{N}} u_{0}(x) d x+\int_{0}^{\infty} \int_{\mathbb{R}^{N}} G(x, t) d x d t .
$$

If $m>1, E_{M_{\infty}}$ is a self-similar solution given by the Barenblatt formula

$$
E_{M_{\infty}}(x, t)=t^{-N k} F\left(x t^{-k}\right) \quad F(x)=\left(\mathcal{C}-k \frac{m-1}{2 m}|x|^{2}\right)_{+}^{1 /(m-1)}
$$

with $k=(N(m-1)+2)^{-1}$. The parameter $\mathcal{C}>0$ is linked with mass $M_{\infty}$ in such a way that $\int_{\mathbb{R}^{N}} E_{M_{\infty}}(x, t) d x=M_{\infty}$ for all $t>0$ (cf. (8), below). For $m=1$, this special solution is simply given by the heat kernel

$$
E_{M_{\infty}}(x, t)=M_{\infty} \frac{e^{-|x|^{2} /(4 t)}}{(4 \pi t)^{N / 2}} .
$$

The proof of (3) for $m>1$ as well as several other results and relevant references concerning the porous media equation (including smoothing properties of solutions) can be found in the review paper by Vázquez [20] and his book [21]. An analogous result for the heat equation $(m=1)$ can be obtained directly from the explicit formula for the solutions, see for instance [8, Thm. 6.1].

The so-called entropy methods allow us to study the convergence of the solutions of Fokker-Planck type equations towards the equilibrium (cf. [3, 18, 1, 14, 12, 11]) in cases where mass is preserved. It is the purpose of this note to show that such methods can also be applied to equations where mass $M=\int_{\mathbb{R}^{N}} v(x, t) d x$ is not conserved in time and eventually diverges as $t \rightarrow \infty$. More precisely, we improve estimate (3) by deriving rates of convergence in $L^{1}\left(\mathbb{R}^{N}\right)$ for the solutions to (1)-(2). Furthermore, these rates are optimal as can be checked on Fokker-Planck type equations without external source terms.

This note is organized as follows. After recalling the known results concerning entropy methods for the homogeneous case in Section 2, we set the problem in the nonhomogeneous case in Section 3 and compute the variation of the relative entropy with respect to some appropriate instantaneous steady state. The last two sections are then devoted to applications of the calculations of Section 3 to the heat and porous medium equations.

Our goal is not to cover the most general case but rather to illustrate the use of relative entropy methods. For simplicity, we shall therefore assume that $m$ is in the range $[1,3 / 2]$. 
2. Homogeneous equations. First, let us recall some known results in the case when the external source term $G(x, t)$ is absent in equation (1). The standard strategy of entropy methods says that, instead of working with (1) directly, the following change of variables (which is a space-time, or time dependent, rescaling) defined by

$$
u(y, s)=e^{N s} v\left(y e^{s}, k\left(e^{s / k}-1\right)\right) \quad \text { with } k=\frac{1}{N(m-1)+2},
$$

transforms the Cauchy problem (1)-(2) with $G \equiv 0$ into the Fokker-Planck equation

$$
u_{s}=\nabla \cdot\left(\nabla u^{m}+y u\right)
$$

while the initial datum is unchanged

$$
u(y, 0)=u_{0}(y)=v_{0}(y)=v(y, 0) .
$$

Equation (5) has the one-parameter family of stationary solutions given by the Barenblatt-Pattle formula

$$
u_{\infty}(y)=\left(\mathcal{C}-\frac{m-1}{2 m}|y|^{2}\right)_{+}^{1 /(m-1)} \quad \text { if } \quad m \neq 1
$$

and by the Gaussian

$$
u_{\infty}(x)=M \frac{e^{-|y|^{2} / 2}}{(2 \pi)^{N / 2}} \quad \text { if } \quad m=1 .
$$

The standard theory that we expose below applies for any $m>(N-1) / N$ if $N=1,2$, and for $m \geq(N-1) / N$ if $N \geq 3$. From now on, we assume that these conditions are always fulfilled. If $m>1$, the constant $\mathcal{C}$ in (6) is chosen in such a way that

$$
\int_{\mathbb{R}^{N}} u_{\infty}(y) d y=M=\int_{\mathbb{R}^{N}} u(y, s) d y
$$

for all $s \geq 0$, which means

$$
M=\mathcal{C}^{\frac{2+N(m-1)}{m-1}}\left(\frac{2 \pi m}{m-1}\right)^{\frac{N}{2}} \frac{\Gamma\left(\frac{m}{m-1}\right)}{\Gamma\left(\frac{N}{2}+\frac{m}{m-1}\right)}
$$

(see [14] for more details). Now, to shorten notations, we define

$$
\sigma(u):= \begin{cases}\frac{u^{m}-u}{m-1} & \text { if } m \neq 1, \\ u \log u & \text { if } m=1 .\end{cases}
$$

According to [16, 17], it is well-known that the entropy,

$$
\Sigma[u(\cdot, s)]:=\int_{\mathbb{R}^{N}}\left[\sigma(u(y, s))+\frac{1}{2}|y|^{2} u(y, s)\right] d y,
$$

plays the role of a Lyapunov functional in the study of the large time behavior of the solutions to (5). First of all, it is decreasing along trajectories:

$$
\frac{d}{d s} \Sigma[u(\cdot, s)]=-\int_{\mathbb{R}^{N}} u\left|y+\nabla \sigma^{\prime}(u)\right|^{2} d y=:-\mathrm{I}[u(\cdot, s)] .
$$

Moreover, the right hand side of (11) controls the relative entropy

$$
\Sigma\left[u \mid u_{\infty}\right]:=\Sigma[u]-\Sigma\left[u_{\infty}\right]
$$


i.e. the difference of the entropy of $u$ and the entropy of the stationary solution $u_{\infty}$, by means of the convex Sobolev inequality:

$$
\Sigma\left[u \mid u_{\infty}\right] \leq \frac{1}{2} \mathrm{I}[u]
$$

for any nonnegative $u \in L^{1}\left(\mathbb{R}^{N}\right)$, provided $m \geq(N-1) / N, N \neq 1,2$. This inequality is the critical Sobolev inequality if $m=(N-1) / N, N \geq 3$, one of Gagliardo-NirenbergSobolev inequalities if $m>(N-1) / N, m \neq 1$ and the logarithmic Sobolev inequality if $m=1$. This can be rewritten as

$$
\int_{\mathbb{R}^{N}}\left(\sigma(u)+\frac{1}{2}|y|^{2} u\right) d y-\mathcal{K} \leq \frac{1}{2} \int_{\mathbb{R}^{N}} u\left|y+\nabla \sigma^{\prime}(u)\right|^{2} d y,
$$

where $\mathcal{K}$ is given in terms of $M=\|u\|_{1}$ by $\mathcal{K}=\int_{\mathbb{R}^{N}}\left(\sigma\left(u_{\infty}\right)+\frac{1}{2}|y|^{2} u_{\infty}\right) d y$, and (6) or (7). Note here the important identities

$$
\sigma^{\prime}\left(u_{\infty}(y)\right)= \begin{cases}(m \mathcal{C}-1) /(m-1)-|y|^{2} / 2 & \text { if } \quad m \neq 1 \\ \log M-\frac{N}{2} \log (2 \pi)-|y|^{2} / 2 & \text { if } \quad m=1\end{cases}
$$

Thus we may rewrite $\Sigma\left[u \mid u_{\infty}\right]$ as

$$
\Sigma\left[u \mid u_{\infty}\right]=\int_{\mathbb{R}^{N}}\left[\sigma(u)-\sigma\left(u_{\infty}\right)-\sigma^{\prime}\left(u_{\infty}\right)\left(u-u_{\infty}\right)\right] d y .
$$

For $m=1$ (so, $\sigma(u)=u \log u$ ), inequality (12) is the logarithmic Sobolev inequality with optimal constants, see $[10,18,19]$. We refer the reader to $[1,14,12]$ for detailed conditions under which (12) can be proved by direct variational methods or by entropy methods for $m>1$, as well as for more general $\sigma$ (also see $[11,7]$ ).

Hence, the Gagliardo-Nirenberg-Sobolev inequality (12) applied to (11) gives an explicit exponential decay of the relative entropy of solutions to (5):

$$
\Sigma\left[u(\cdot, s) \mid u_{\infty}\right] \leq \Sigma\left[u_{0} \mid u_{\infty}\right] \cdot e^{-2 s} .
$$

The next step is to measure the exponential convergence of $u(\cdot, t)$ towards $u_{\infty}$ in terms of a norm. This can be done using the Csiszár-Kullback inequality, for $m=1$, as follows. Lemma $1([13,15])$. Let $\phi, \phi_{0} \in L_{+}^{1}\left(\mathbb{R}^{N}, d \mu\right)$. Assume that $\sigma$ is a convex function on $\mathbb{R}^{+}$ such that $0=\sigma(1)=\min _{\mathbb{R}^{+}} \sigma$ and

$$
K=\min \left\{\inf _{t \in[0,1]} \sigma^{\prime \prime}(t), \inf _{\substack{t \geq 0 \\ \theta \in[0,1]}} \sigma^{\prime \prime}(1+\theta t)(1+t)\right\}>0
$$

is positive. Then

$$
\left\|\phi-\phi_{0}\right\|_{L^{1}\left(\mathbb{R}^{N}, d \mu\right)}^{2} \leq \frac{4 \mathcal{M}}{K} \int_{\mathbb{R}^{N}} \sigma\left(\frac{\phi}{\phi_{0}}\right) \phi_{0} d \mu
$$

with $\mathcal{M}=\max \left\{\|\phi\|_{L^{1}\left(\mathbb{R}^{N}, d \mu\right)},\left\|\phi_{0}\right\|_{L^{1}\left(\mathbb{R}^{N}, d \mu\right)}\right\}$.

Inequality (14) was introduced in $[13,15]$. We refer the reader to $[2,14,9]$ for a proof of Lemma 1 and some extensions.

If $m=1$, one combines inequalities (14) with (13) to obtain

$$
\left\|u(\cdot, s)-u_{\infty}\right\|_{1}^{2} \leq 4 M \Sigma\left[u_{0} \mid u_{\infty}\right] \cdot e^{-2 s}
$$


for all $t \geq 0$. When $m>1$, several approaches are possible. One can, for instance, control a weighted $L^{1}$-norm, see, e.g., $[14,7]$. With some additional work, one can also obtain a control of the usual $L^{1}$-norm like in the case $m=1$ as it was done in [12]. Below, see Proposition (2) in Section 5, we recall some of these results and give a self-contained and slightly simplified proof.

Finally, going back to the original problem (1)-(2) with $G \equiv 0$, via the time-dependent rescaling (4), one shows that for each $m \in[1,2]$

$$
\left\|v(\cdot, t)-E_{M}(\cdot, t)\right\|_{1}^{2} \leq C\left(1+\frac{t}{k}\right)^{-2 k} \quad \text { with } \quad k=\frac{1}{N(m-1)+2}
$$

for all $t>0$ and a constant $\mathrm{C}$ depending only on $M, \Sigma\left[u_{0} \mid u_{\infty}\right]$, and $m$.

3. Nonhomogeneous equations. In the case of the Cauchy problem (1)-(2) with nonzero external source terms, calculations are similar. We use the space-time change of variables analogous to that in Section 2:

$$
\begin{aligned}
& u(y, s)=e^{N s} v\left(y e^{s}, k\left(e^{s / k}-1\right)\right), \quad k=\frac{1}{N(m-1)+2}, \\
& F(y, s)=e^{(N+2) s} G\left(y e^{s}, k\left(e^{s / k}-1\right)\right),
\end{aligned}
$$

which transforms the Cauchy problem (1)-(2) into

$$
\begin{aligned}
& u_{s}=\nabla \cdot\left(\nabla u^{m}+y u\right)+F(y, s), \\
& u(y, 0)=u_{0}(y)=v_{0}(y) .
\end{aligned}
$$

The main assumption of this note reads as follows.

Assumption 1. Let $m \in[1,2]$. The nonnegative functions $u_{0}$ and $F$ satisfy

$$
\begin{aligned}
& u_{0} \in L^{1} \cap L^{m}\left(\mathbb{R}^{N}\right), \quad|y|^{2} u_{0} \in L^{1}\left(\mathbb{R}^{N}\right), \\
& F \in L^{1}\left(\mathbb{R}^{N} \times[0, T]\right) \cap L^{1}\left([0, T], L^{1 /(2-m)}\left(\mathbb{R}^{N}\right)\right)
\end{aligned}
$$

for all $T>0\left(\right.$ in the case $m=2, L^{1 /(2-m)}\left(\mathbb{R}^{N}\right)$ means $\left.L^{\infty}\left(\mathbb{R}^{N}\right)\right)$. If $m=1$, we assume moreover that

$$
u_{0} \log u_{0} \in L^{1}\left(\mathbb{R}^{N}\right) \quad \text { and } \quad F \log F \in L^{1}\left(\mathbb{R}^{N} \times[0, T]\right)
$$

for all $T>0$.

This assumption implies, in particular, that the mass of the solution to (16)-(17)

$$
M(s)=\int_{\mathbb{R}^{N}} u(y, s) d y=\int_{\mathbb{R}^{N}} u_{0}(y) d y+\int_{0}^{s} \int_{\mathbb{R}^{N}} F(y, s) d y d s
$$

is positive for all $s \geq 0$.

Under the change of variables $(15)$, with $t=k\left(e^{s / k}-1\right), x=y e^{s}$, mass is preserved:

$$
M(s)=\int_{\mathbb{R}^{N}} u(y, s) d y=\int_{\mathbb{R}^{N}} v(x, t) d x=: \bar{M}(t) .
$$


Define the family of the instantaneous steady states or local Gibbs states for $m \neq 1$ by:

$$
u_{\infty}(y, s)=\left(\mathcal{C}(s)-\frac{m-1}{2 m}|y|^{2}\right)_{+}^{1 /(m-1)},
$$

so that the choice of the function $\mathcal{C}(s)$ guarantees

$$
\int_{\mathbb{R}^{N}} u_{\infty}(y, s) d y=M(s) \text { for all } s \geq 0
$$

Hence, $\mathcal{C}(s)$ is given in terms of $M(s)$ by the formula (8). If $m=1$, we simply put

$$
u_{\infty}(y, s)=M(s) \frac{e^{-|y|^{2} / 2}}{(2 \pi)^{N / 2}} .
$$

Now, in the case of solutions to the nonhomogeneous equation (16), we do not expect the entropy $\Sigma[u(\cdot, s)]$ defined in (10) to decrease because of the presence of the external source term $F(y, s)$. Let $\sigma$ be given by (9). Our goal is to show, however, that the relative entropy

$$
\begin{aligned}
\Sigma(s) & =\Sigma\left[u(\cdot, s) \mid u_{\infty}(\cdot, s)\right]:=\Sigma[u(\cdot, s)]-\Sigma\left[u_{\infty}(\cdot, s)\right] \\
& =\int_{\mathbb{R}^{N}}\left[\sigma(u(y, s))-\sigma\left(u_{\infty}(y, s)\right)-\sigma^{\prime}\left(u_{\infty}(y, s)\right)\left(u(y, s)-u_{\infty}(y, s)\right)\right] d y
\end{aligned}
$$

still can be used to show the convergence of solutions towards the family of instantaneous steady states defined in (19) and (21). The crucial estimate is contained in the following proposition. We state it here at a formal level and will explain in Sections 4 and 5 how to extend it to more general solutions corresponding to initial data satisfying Assumption 1.

Proposition 1. Let $u$ be a sufficiently smooth solution to problem (16)-(17). Then

$$
\frac{d}{d s} \Sigma\left[u \mid u_{\infty}\right]=-\int_{\mathbb{R}^{N}} u\left|\nabla \sigma^{\prime}(u)-\nabla \sigma^{\prime}\left(u_{\infty}\right)\right|^{2} d y+\int_{\mathbb{R}^{N}}\left[\sigma^{\prime}(u)-\sigma^{\prime}\left(u_{\infty}\right)\right] F d y .
$$

Proof. The derivation with respect to $s$ of $\Sigma(s)=\Sigma\left[u(\cdot, s) \mid u_{\infty}(\cdot, s)\right]$ gives

$$
\begin{aligned}
\frac{d \Sigma}{d s} & =\frac{d}{d s} \int_{\mathbb{R}^{N}}\left[\sigma(u)-\sigma\left(u_{\infty}\right)-\sigma^{\prime}\left(u_{\infty}\right)\left(u-u_{\infty}\right)\right] d y \\
& =\int_{\mathbb{R}^{N}}\left[\sigma^{\prime}(u)-\sigma^{\prime}\left(u_{\infty}\right)\right] u_{s} d y-\int_{\mathbb{R}^{N}}\left(\sigma^{\prime}\left(u_{\infty}\right)\right)_{s}\left(u-u_{\infty}\right) d y .
\end{aligned}
$$

Because of (19), the second term can be written as

$$
\int_{\mathbb{R}^{N}}\left(\sigma^{\prime}\left(u_{\infty}\right)\right)_{s}\left(u-u_{\infty}\right) d y=\frac{d \mathcal{C}}{d s} \int_{\mathbb{R}^{N}}\left(u-u_{\infty}\right) d y=0,
$$

where $\mathcal{C}=\mathcal{C}(s)$ is the function of $M(s)$ which appears in (19) if $m \neq 1$ and $d \mathcal{C} / d s=$ $-M^{\prime}(s) / M(s)$ if $m=1$. Using (16) and integrating by parts, the first term on the right hand side of $(24)$ is

$$
\begin{gathered}
\int_{\mathbb{R}^{N}}\left[\sigma^{\prime}(u)-\sigma^{\prime}\left(u_{\infty}\right)\right] u_{s} d y=-\int_{\mathbb{R}^{N}} \nabla\left[\sigma^{\prime}(u)-\sigma^{\prime}\left(u_{\infty}\right)\right]\left(\nabla u^{m}+y u\right) d y \\
+\int_{\mathbb{R}^{N}}\left[\sigma^{\prime}(u)-\sigma^{\prime}\left(u_{\infty}\right)\right] F d y
\end{gathered}
$$

which proves the result using $\nabla u^{m}+y u=u\left[\nabla \sigma^{\prime}(u)-\nabla \sigma^{\prime}\left(u_{\infty}\right)\right]$. 
REMARK 1. If we integrate equation (23) with respect to $s$, all quantities will be well defined and, as a consequence, $u$ and $|y|^{2} u$ will be bounded respectively in $L^{\infty}\left(\mathbb{R}^{+}, L^{1} \cap\right.$ $\left.L^{m}\left(\mathbb{R}^{N}\right)\right)$ and $L^{\infty}\left(\mathbb{R}^{+}, L^{1}\left(\mathbb{R}^{N}\right)\right)$. Since $u \mapsto \Sigma\left[u \mid u_{\infty}\right]$ and, for $1 \leq m \leq 3 / 2, u \mapsto$ $\int_{\mathbb{R}^{N}} u\left|\nabla \sigma^{\prime}(u)\right|^{2} d y$ are convex, we can then easily extend (23) to less regular functions by a density argument. Note that the convexity of $\Sigma\left[u \mid u_{\infty}\right]$ holds under the constraint that for any $s \geq 0$,

$$
\int_{\mathbb{R}^{N}} u(y, s) d y=M(s)=\int_{\mathbb{R}^{N}} u_{\infty}(y, s) d y .
$$

Here, the restriction $m \leq 3 / 2$ in this reasoning comes from the fact that we use the convexity property of $u \mapsto \int_{\mathbb{R}^{N}}\left|\nabla u^{\gamma}\right|^{2} d y$, which holds true if and only if $m-1 / 2=\gamma \in$ $[1 / 2,1]$ (see $[5,6])$. For $m>3 / 2$, a further analysis of the regularity of the solutions would be required to proceed as in the homogeneous case, cf. [12, 14].

REMARK 2. It is remarkable that even when mass varies, $\Sigma\left[u \mid u_{\infty}\right]$ is still a good Lyapunov function. Actually this holds because the constraint (25) is taken into account in the definition of $u_{\infty}$. For several reasons, it makes sense to write that $\Sigma\left[u \mid u_{\infty}\right]$ is the relative entropy of $u$ with respect to $u_{\infty}$. See [4] for more comments on this type of issues.

The next step is to combine equality (23) with the generalized Sobolev inequality (12) and to find an estimate of the second term on the right-hand side of (23) by a quantity independent of $u$. This procedure is realized in the next two sections for the heat equation $(m=1)$ and for the porous medium equation with $1<m \leq 3 / 2$, separately.

4. Application to the heat equation. Consider first the nonhomogeneous heat equation

$$
v_{t}=\Delta v+G(x, t), \quad x \in \mathbb{R}^{N}, t>0 .
$$

By the time dependent rescaling (15) with $m=1$, we have

$$
\begin{aligned}
& u(y, s)=e^{N s} v\left(y e^{s}, \frac{1}{2}\left(e^{2 s}-1\right)\right), \\
& F(y, s)=e^{(N+2) s} G\left(y e^{s}, \frac{1}{2}\left(e^{2 s}-1\right)\right) .
\end{aligned}
$$

Hence, equation (26) is transformed into a Fokker-Planck equation with the additional external source term $F$

$$
u_{s}=\nabla \cdot(\nabla u+y u)+F(y, s)
$$

This equation is supplemented with the initial condition

$$
u(y, 0)=u_{0}(y)
$$

Let us recall that the stationary steady state $u_{\infty}$ of the homogeneous problem $\nabla \cdot\left(\nabla u_{\infty}+\right.$ $\left.y u_{\infty}\right)=0$ with mass $M(s)$ is given by the formula $(21)$, where mass $M(s)$ of the solution is defined by (18):

$$
u_{\infty}(y, s)=M(s) \bar{u}(y), \quad \bar{u}(y)=\frac{e^{-|y|^{2} / 2}}{(2 \pi)^{N / 2}} .
$$

Our main result on the large time behavior of solutions to (29)-(30) reads as follows. 
Theorem 1. Suppose that $u_{0}, F(\cdot, s) \in L^{1}\left(\mathbb{R}^{N},\left(1+|y|^{2}\right) d y\right)$ for every $s \geq 0$ satisfy Assumption 1. Then for all $s \geq 0$, the solution of problem (29)-(30) satisfies the inequality

$$
\begin{aligned}
& \left\|u(s, \cdot)-u_{\infty}(s, \cdot)\right\|_{1}^{2} \\
& \leq 4 M(s) e^{-2 s}\left[\Sigma\left[u_{0} \mid u_{\infty}(0, \cdot)\right]+\int_{0}^{s} e^{2 \tau} \int_{\mathbb{R}^{N}} F \log \left(\frac{F}{\left(\int_{\mathbb{R}^{N}} F d y\right) \bar{u}}\right) d y d \tau\right] .
\end{aligned}
$$

Proof. For $m=1$, the relative entropy of the solution $u$ with respect to $u_{\infty}$ given by (22) takes the form

$$
\Sigma(s):=\Sigma\left[u(\cdot, s) \mid u_{\infty}(\cdot, s)\right]=\int_{\mathbb{R}^{N}} u(y, s) \log \left(\frac{u(y, s)}{u_{\infty}(y, s)}\right) d y .
$$

Hence, it follows from Proposition 1 that

$$
\frac{d \Sigma}{d s}=-\int_{\mathbb{R}^{N}} u\left|\frac{\nabla u}{u}+y\right|^{2} d y+\int_{\mathbb{R}^{N}} F \log \left(\frac{u}{M(s) \bar{u}}\right) d y .
$$

Next, we use the logarithmic Sobolev inequality (12), which in this case reduces to

$$
\Sigma\left[u \mid u_{\infty}\right] \leq \frac{1}{2} \int_{\mathbb{R}^{N}} u\left|\frac{\nabla u}{u}+y\right|^{2} d y
$$

and obtain

$$
\frac{d \Sigma}{d s} \leq-2 \Sigma\left[u(\cdot, s) \mid u_{\infty}(\cdot, s)\right]+\int_{\mathbb{R}^{N}} F \log \left(\frac{u}{u_{\infty}}\right) d y .
$$

Finally, after multiplying this inequality by $e^{2 s}$ and integrating with respect to $s$, we arrive at

$$
\Sigma(s) \leq e^{-2 s}\left[\Sigma(0)+\int_{0}^{s} e^{2 \tau}\left(\int_{\mathbb{R}^{N}} F(y, \tau) \log \left(\frac{u(y, \tau)}{u_{\infty}(y, \tau)}\right) d y\right) d \tau\right] .
$$

We are going to estimate the second term of the right hand side of this inequality using the lemma formulated below.

Lemma 2. Assume that $f$ and $w$ are two nonnegative integrable functions on $\mathbb{R}^{N}$. Then

$$
\int_{\mathbb{R}^{N}} f \log \left(\frac{w}{\|w\|_{1}}\right) d y \leq \int_{\mathbb{R}^{N}} f \log \left(\frac{f}{\|f\|_{1}}\right) d y
$$

Proof. Apply the Jensen inequality to the convex function $\varphi \mapsto \varphi \log \varphi$ and the probability measure $d \mu=\|w\|_{1}^{-1} w d y$ with $\varphi=f / w$ :

$$
\begin{aligned}
\int_{\mathbb{R}^{N}} f \log \left(\frac{f}{w}\right) d y & =\|w\|_{1} \int_{\mathbb{R}^{N}} \varphi \log \varphi d \mu \\
& \geq\|w\|_{1}\left(\int_{\mathbb{R}^{N}} \varphi d \mu\right) \log \left(\int_{\mathbb{R}^{N}} \varphi d \mu\right)=\|f\|_{1} \log \left(\frac{\|f\|_{1}}{\|w\|_{1}}\right) .
\end{aligned}
$$

Note that the two sides of (32) may be infinite.

We come back to the proof of Theorem 1 . If we write

$$
\int_{\mathbb{R}^{N}} F \log \left(\frac{u}{M \bar{u}}\right) d y=\int_{\mathbb{R}^{N}} F \log \left(\frac{u}{M}\right) d y-\int_{\mathbb{R}^{N}} F \log \bar{u} d y
$$

and apply Lemma 2 with $f=F$ and $w=u$ to the first term of the right hand side, then the result easily follows using the Csiszár-Kullback inequality stated in Lemma 1. 
REMARK 3. The result of Lemma 2 is a limit case of Hölder's inequality. Let $q_{0}>1$ and assume that both $f$ and $w$ belong to $L^{1} \cap L^{q_{0}}\left(\mathbb{R}^{N}\right)$. Then it follows from Hölder's inequality that

$$
\int_{\mathbb{R}^{N}} w^{q-1} f d y \leq\left(\int_{\mathbb{R}^{N}} w^{q} d y\right)^{\frac{q-1}{q}}\left(\int_{\mathbb{R}^{N}} f^{q} d y\right)^{1 / q}
$$

for every $1 \leq q \leq q_{0}$. Note that if $q=1$ this inequality reduces to $\int_{\mathbb{R}^{N}} f=\int_{\mathbb{R}^{N}} f$, which immediately implies that

$$
\int_{\mathbb{R}^{N}} w^{q-1} f d y-\int_{\mathbb{R}^{N}} f d y \leq\left(\int_{\mathbb{R}^{N}} w^{q} d y\right)^{\frac{q-1}{q}}\left(\int_{\mathbb{R}^{N}} f^{q} d y\right)^{1 / q}-\int_{\mathbb{R}^{N}} f d y .
$$

Dividing both sides by $q-1$ and taking the limit as $q \rightarrow 1$, we obtain inequality (32). The assumption that $f, w \in L^{1} \cap L^{q_{0}}\left(\mathbb{R}^{N}\right)$ is easily removed by a density argument, which provides an alternative proof of Lemma 2 .

According to (27)-(28), the results of Theorem 1 written in terms of the original coordinates give intermediate asymptotics results as follows.

COROLlaRY 1. Under the same assumptions as in Theorem 1, if $u$ and $v$ are related by (27), and $F$ and $G$ by (28), then for any $t \geq 0$,

$\left\|v(\cdot, t)-v_{\infty}(\cdot, t)\right\|_{1}^{2} \leq \frac{4 \bar{M}(t)}{1+2 t}\left[\Sigma\left[v_{0} \mid v_{\infty}(\cdot, 0)\right]+\int_{0}^{t}(1+2 \tau) \int_{\mathbb{R}^{N}} G \log \left(\frac{\bar{M}(\tau) G}{\left(\int_{\mathbb{R}^{N}} G d x\right) v_{\infty}}\right) d x d \tau\right]$.

where $\bar{M}(t)=\int_{\mathbb{R}^{N}} v(x, t) d x$ and

$$
v_{\infty}(x, t)=\frac{\bar{M}(t)}{(1+2 t)^{N / 2}} \bar{u}\left(\frac{x}{\sqrt{1+2 t}}\right), \quad \bar{u}(x)=\frac{e^{-|x|^{2} / 2}}{(2 \pi)^{N / 2}} .
$$

Rather than writing abstract conditions on $G$ in order to guarantee that $\left\|\left(v-v_{\infty}\right)(\cdot, t)\right\|_{1}$ converges to 0 , let us simply formulate two examples which illustrate both Theorem 1 and Corollary 1.

EXAMPLE 1. Let us look at inequality (31) in the case of external source terms of the form $F(y, s)=g(x) f(s)$ with suitably chosen $g$ and $f$. For such a choice of $F$, we have

$$
\int_{\mathbb{R}^{N}} F(y, \tau) \log \left[\frac{F(y, \tau)}{\left(\int_{\mathbb{R}^{N}} F d y\right) \bar{u}(y)}\right] d y=f(\tau) \int_{\mathbb{R}^{N}} g(y) \log \left[\frac{g(y)}{\left(\int_{\mathbb{R}^{N}} g d y\right) \bar{u}(y)}\right] d y .
$$

If the second factor on the right-hand side is finite, the problem is therefore reduced to understand the behavior as $s \rightarrow \infty$ of the quantity

$$
e^{-2 s} \int_{0}^{s} e^{2 \tau} f(\tau) d \tau
$$

Choosing, e.g., $f(s)=e^{-\kappa s}$ for some $\kappa>0$, we immediately obtain

$$
e^{-2 s} \int_{0}^{s} e^{2 \tau} e^{-\kappa \tau} d \tau=\frac{e^{-\kappa s}-e^{-2 s}}{2-\kappa}
$$

In this case, mass $M(s)$ is bounded uniformly in $s$ according to (18) and Theorem 1 applies:

$$
\left\|u(\cdot, s)-u_{\infty}(\cdot, s)\right\|_{1}^{2} \leq C\left(e^{-2 s}+e^{-\kappa s}\right) \quad \forall s \geq 0
$$


for some positive constant $C$. Now, we may come back to the solutions of the nonhomogeneous heat equation (26) via the rescaling (27)-(28) and reformulate (33) as

$$
\left\|v(\cdot, t)-v_{\infty}(\cdot, t)\right\|_{1}^{2} \leq C\left[(1+t)^{-1}+(1+t)^{-2 \kappa}\right] .
$$

EXAmple 2. As a second example, let us consider $F(y, s)=g(y)(1+s)^{-\alpha}$ for some $\alpha>0$. A direct calculation shows that

$$
e^{-2 s} \int_{0}^{s} e^{2 \tau}(1+\tau)^{-\alpha} d \tau \leq C(1+s)^{-\alpha}
$$

for a constant $C>0$ and all $s>0$, and consequently, by Theorem 1 ,

$$
\left\|u(\cdot, s)-u_{\infty}(\cdot, s)\right\|_{1}^{2} \leq C M(s)(1+s)^{-\alpha} .
$$

for some constant $C>0$. Here, $\alpha \in(0,1]$ is to the most interesting case because

$$
\begin{aligned}
M(s) & =\int_{\mathbb{R}^{N}} u_{0}(y) d y+\int_{\mathbb{R}^{N}} g(y) d y \int_{0}^{s}(1+\tau)^{-\alpha} d \tau \\
& =\left\|u_{0}\right\|_{1}+\|g\|_{1} \frac{(1+s)^{1-\alpha}-1}{1-\alpha} \rightarrow \infty
\end{aligned}
$$

as $s \rightarrow \infty$. However, $u-u_{\infty}$ still tends towards 0 in the $L^{1}$-norm provided $\alpha>1 / 2$.

We can again reformulate inequality (34) for solutions of the nonhomogeneous heat equation (26)

$$
\left\|v(\cdot, t)-v_{\infty}(\cdot, t)\right\|_{1}^{2} \leq C \bar{M}(t)(\log t)^{-\alpha} \leq C(\log t)^{1-2 \alpha} \rightarrow 0 \quad \text { as } t \rightarrow \infty
$$

for $\alpha>1 / 2$, since mass $\bar{M}(t)$ is of order $O\left((\log t)^{1-\alpha}\right)$ as $t \rightarrow \infty$. Hence, by our method, we can extend in some cases the result formulated in (3) to source terms $G=G(x, t)$ for which $M_{\infty}=\lim _{t \rightarrow \infty} \int_{\mathbb{R}^{N}} v(x, t) d x=\infty$.

5. Application to the porous medium equation. In this section, we deal with the nonlinear Cauchy problem (16)-(17) with $m>1$ for which the relative entropy of the solution $u$ with respect to $u_{\infty}$ given by $(22)$ takes the form

$$
\begin{aligned}
\Sigma(s) & :=\Sigma\left[u(\cdot, s) \mid u_{\infty}(\cdot, s)\right]=\Sigma[u(\cdot, s)]-\Sigma\left[u_{\infty}(\cdot, s)\right] \\
& =\frac{1}{m-1} \int_{\mathbb{R}^{N}}\left[u^{m}-u_{\infty}^{m}-\frac{m-1}{2}|x|^{2}\left(u-u_{\infty}\right)\right] d y,
\end{aligned}
$$

where $u_{\infty}(y, s)$ is given by $(19)$.

The main result on the convergence of $u(s)$ toward the family of instantaneous steady states is contained in the next theorem. As in the case of the linear heat equation, one can reformulate this result for the original problem (1)-(2) going back via the rescaling (15). Theorem 2. Let $m \in\left(1, \frac{3}{2}\right]$. Assume that $u_{0}$ and $F$ satisfy Assumption 1 . Let $u$ be the solution to (16)-(17) with $M(s)$ defined in (18) and $u_{\infty}(s, y)$ given by (19)-(20). Suppose moreover that $M_{\infty} \equiv \sup _{s>0} M(s)$ is finite.

Then there exists a constant $C>0$ depending on $M_{\infty}$ but independent of s such that

$$
\left\|u(s, \cdot)-u_{\infty}(s, \cdot)\right\|_{1}^{2} \leq C e^{-2 s}\left[\Sigma\left[u_{0} \mid u_{\infty}(0, \cdot)\right]^{\frac{1}{m}}+\frac{1}{m} \int_{0}^{s} e^{\frac{2}{m} \tau}\|F(\cdot, \tau)\|_{m} d \tau\right]^{m}
$$

for all $s \geq 0$. 
Here, we assume that $m \in(1,3 / 2]$ because of the convexity argument mentioned in Remark 1. This assumption plays also the crucial role in the proof of Lemma 3, below.

Before proving Theorem 2, we need some preliminary estimates.

Lemma 3. Assume that $p \geq 3$ and let $\mu$ be a positive bounded measure. Then for any nonnegative $w \in L^{p}(d \mu)$,

$$
\int|w-1|^{p} d \mu \leq(p-1) \int\left[w^{p}-1-\frac{p}{p-1}\left(w^{p-1}-1\right)\right] d \mu .
$$

Proof. Let $f(w):=w^{p}-1-\frac{p}{p-1}\left(w^{p-1}-1\right)-\frac{1}{p-1}|w-1|^{p}$. A straightforward computation gives

$$
\begin{aligned}
& f^{\prime}(w)=p w^{p-2}(w-1)-\frac{p}{p-1}|w-1|^{p-2}(w-1), \\
& f^{\prime \prime}(w)=p w^{p-3}[(p-1)(w-1)+1]-p|w-1|^{p-2} .
\end{aligned}
$$

First of all, $f(1)=f^{\prime}(1)=0$ and

$$
\frac{1}{p} f^{\prime \prime}(w) \geq(w-1)^{p-3}[(p-1)(w-1)+1]-(w-1)^{p-2} \geq(p-2)(w-1)^{p-2}
$$

for any $w \geq 1$. Thus $f$ is convex and therefore nonnegative on $(1,+\infty)$.

On $(0,1), f^{\prime \prime}$ is increasing. Since $\lim _{w \rightarrow 0+} f^{\prime \prime}(w)<0$, there exists $w_{*}$ such that $f$ is concave on $\left(0, w_{*}\right)$ and convex on $\left(w_{*}, 1\right)$. Thus the minimum of $f$ on $(0,1)$ is achieved either at $w=0$ or at $w=1$. Since $f(0)=f(1)=0$, this proves that $f$ is also nonnegative on $(0,1)$.

Corollary 2. Let $F$ and $u$ be two nonnegative functions respectively in $L^{m}\left(\mathbb{R}^{N}\right)$ and $L^{1} \cap L^{m}\left(\mathbb{R}^{N}\right)$ and consider $u_{\infty}$ given by (6) such that $\|u\|_{1}=\left\|u_{\infty}\right\|_{1}$. If $m \in(1,3 / 2]$, then

$$
\int_{\mathbb{R}^{N}}\left|u^{m-1}-u_{\infty}^{m-1}\right| F d y \leq \Sigma\left[u \mid u_{\infty}\right]^{\frac{m-1}{m}}\|F\|_{m}
$$

Proof. Let $w:=v^{m-1}, p:=m /(m-1)$ and $d \mu:=u_{\infty}^{m} d y$ in Lemma 3. Hence, inequality (36) can be rewritten as

$$
\int_{\mathbb{R}^{N}}\left|v^{m-1}-1\right|^{\frac{m}{m-1}} u_{\infty}^{m} d y \leq \frac{1}{m-1} \int_{\mathbb{R}^{N}}\left[v^{m}-1-m(v-1)\right] u_{\infty}^{m} d y .
$$

If we let $v=u / u_{\infty}$, this means

$$
\begin{aligned}
\int_{\operatorname{supp}\left(u_{\infty}\right)}\left|u^{m-1}-u_{\infty}^{m-1}\right| \frac{m}{m-1} d y \\
\quad \leq \frac{1}{m-1} \int_{\operatorname{supp}\left(u_{\infty}\right)}\left[u^{m}-u_{\infty}^{m}-m u_{\infty}^{m-1}\left(u-u_{\infty}\right)\right] d y \\
\quad=\frac{1}{m-1} \int_{\operatorname{supp}\left(u_{\infty}\right)}\left[u^{m}-u_{\infty}^{m}-m\left(\mathcal{C}-\frac{m-1}{2 m}|y|^{2}\right)\left(u-u_{\infty}\right)\right] d y .
\end{aligned}
$$

On the other hand, since $(m-1)^{-1}>1$ and, on the set $\operatorname{supp}\left(u_{\infty}\right)^{c}$, we have

$$
\mathcal{C}-\frac{m-1}{2 m}|y|^{2} \leq 0
$$


as well as $u_{\infty}=0$, we may proceed in the most direct way as follows:

$$
\begin{aligned}
& \int_{\operatorname{supp}\left(u_{\infty}\right)^{c}}\left|u^{m-1}-u_{\infty}^{m-1}\right| \frac{m}{m-1} d y \\
& \quad \leq \frac{1}{m-1} \int_{\operatorname{supp}\left(u_{\infty}\right)^{c}} u^{m} d y \\
& \quad \leq \frac{1}{m-1} \int_{\operatorname{supp}\left(u_{\infty}\right)^{c}}\left[u^{m}-u_{\infty}^{m}-m\left(\mathcal{C}-\frac{m-1}{2 m}|y|^{2}\right)\left(u-u_{\infty}\right)\right] d y .
\end{aligned}
$$

Summing up both estimates we obtain

$$
\int_{\mathbb{R}^{N}}\left|u^{m-1}-u_{\infty}^{m-1}\right|^{\frac{m}{m-1}} d y \leq \Sigma\left[u \mid u_{\infty}\right]
$$

Hence the proof is completed by using Hölder's inequality as follows:

$$
\int_{\mathbb{R}^{N}}\left|u^{m-1}-u_{\infty}^{m-1}\right| F d y \leq\left[\int_{\mathbb{R}^{N}}\left|u^{m-1}-u_{\infty}^{m-1}\right|^{\frac{m}{m-1}} d y\right]^{\frac{m-1}{m}}\|F\|_{m} .
$$

REMARK 4. In Corollary 2, the exponent $p=m /(m-1)$ is the Hölder conjugate of $m$. Thus the assumption $m \leq 3 / 2$ is equivalent to $p \geq 3$, which is used in the proof of Lemma 3.

In the next lemma, we state and prove an inequality of Csiszár-Kullback type which differs from the one recalled in Lemma 1 . The results formulated below are contained in [12]. Here, however, we give direct and elementary proofs. Recall that, in this section, the relative entropy $\Sigma\left[u \mid u_{\infty}\right]$ is given by formula (35).

Proposition 2. Assume that $1<m \leq 2$. Let $u$ be a nonnegative function in $L^{1}\left(\mathbb{R}^{N}\right)$ such that $\Sigma\left[u \mid u_{\infty}\right] \leq \Sigma_{0}$ is finite. Then there exists a positive constant $C$, which only depends on $\Sigma_{0}$ and $M=\int_{\mathbb{R}^{N}} u d y$, such that

$$
\left\|u-u_{\infty}\right\|_{L^{1}\left(\mathbb{R}^{N}\right)}^{2} \leq C \Sigma\left[u \mid u_{\infty}\right] .
$$

Proof. Let $B=B(0, R)$ be the support of $u_{\infty}$. On $B$, let $v:=u / u_{\infty}$, so that

$$
(m-1) \Sigma\left[u \mid u_{\infty}\right]=\int_{B}\left[v^{m}-1-m(v-1)\right] u_{\infty}^{m} d y+\int_{B^{c}}\left[u^{m}+\frac{m-1}{2}|y|^{2} u\right] d y .
$$

1) On $B^{c}$, using the last term of the right hand side of the above equation, for $C_{1}:=$ $\frac{2}{m-1} \frac{1}{R^{2}}$ we get

$$
\int_{B^{c}}\left[u^{m}+\frac{m-1}{2}|y|^{2} u\right] d y \geq C_{1} \int_{|y|>R} u d y=C_{1}\left\|u-u_{\infty}\right\|_{L^{1}\left(B^{c}\right)} .
$$

2) Using a Taylor expansion at order 2, we get

$$
v^{m}-1-m(v-1)=\frac{1}{2} m(m-1)(\tau+(1-\tau) v)^{m-2}
$$

for some function $\tau$ with values in $(0,1)$. If $v>1$, then

$$
(\tau+(1-\tau) v)^{m-2} \geq v^{m-2} .
$$


By Hölder's inequality, on $\omega:=\{y \in B: v(y)>1\}$,

$$
\begin{aligned}
\int_{\omega}\left|u-u_{\infty}\right| d y & =\int_{\omega}|v-1| u_{\infty} d y \\
& =\int_{\omega}\left(|v-1|^{2} v^{m-2} u_{\infty}^{m}\right)^{\frac{1}{m}} \cdot\left(\frac{|v-1|}{v}\right)^{1-\frac{2}{m}} d y \\
& \leq\left(\int_{\omega}|v-1|^{2} v^{m-2} u_{\infty}^{m} d y\right)^{1 / m} \frac{1}{N}\left|S^{N-1}\right| R^{N} .
\end{aligned}
$$

This proves that

$$
\begin{aligned}
\int_{\omega}\left[v^{m}-1-m(v-1)\right] u_{\infty}^{m} d y & \geq \frac{m}{2}(m-1) \int_{\omega}|v-1|^{2} v^{m-2} u_{\infty}^{m} d y \\
& \geq C_{2}\left\|u-u_{\infty}\right\|_{L^{1}(\omega)}^{m}
\end{aligned}
$$

for some positive constant $C_{2}$.

3) Similarly on $B \backslash \omega$, that is for $0<v<1$,

$$
(\tau+(1-\tau) v)^{m-2} \geq 1
$$

By the Cauchy-Schwarz inequality,

$$
\left\|u-u_{\infty}\right\|_{L^{1}(B \backslash \omega)}^{2}=\left(\int_{B \backslash \omega}|v-1| u_{\infty} d y\right)^{2} \leq \int_{B \backslash \omega}|v-1|^{2} u_{\infty}^{m} d y \cdot \int_{B \backslash \omega} u_{\infty}^{2-m} d y
$$

so that

$$
\begin{aligned}
\int_{B \backslash \omega}\left[v^{m}-1-m(v-1)\right] u_{\infty}^{m} d y & \geq \frac{m}{2}(m-1) \int_{B \backslash \omega}|v-1|^{2} u_{\infty}^{m} d y \\
& \geq C_{3}\left\|u-u_{\infty}\right\|_{L^{1}(B \backslash \omega)}^{2}
\end{aligned}
$$

for some positive constant $C_{3}$.

Let $t_{1}:=\int_{|y|>R} u d y, t_{2}:=\left\|u-u_{\infty}\right\|_{L^{1}(\omega)}$ and $t_{3}:=\left\|u-u_{\infty}\right\|_{L^{1}(B \backslash \omega)}$. Since

$$
\max _{i=1,2,3} t_{i} \leq\left\|u-u_{\infty}\right\|_{L^{1}\left(\mathbb{R}^{N}\right)}
$$

is bounded from above by $2 M$, the quantity $C_{1} t_{1}+C_{2} t_{2}^{m}+C_{3} t^{2}$ is bounded from below by $2(m-1) C\left(t_{1}^{2}+t_{2}^{2}+t_{3}^{2}\right) \geq(m-1) C\left(t_{1}+t_{2}+t_{3}\right)^{2}$ on $(0,2 M)$ with $C:=$ $\min \left\{C_{1} /(2 M), C_{2} /(2 M)^{2-m}, C_{3}\right\} /(2(m-1))$.

Proof of Theorem 2. It follows from Proposition 1 that

$$
\frac{d}{d s} \Sigma\left[u \mid u_{\infty}\right]=-\int_{\mathbb{R}^{N}} u\left|\nabla \sigma^{\prime}(u)-\nabla \sigma^{\prime}\left(u_{\infty}\right)\right|^{2} d y+\frac{m}{m-1} \int_{\mathbb{R}^{N}}\left[u^{m-1}-u_{\infty}^{m-1}\right] F d y .
$$

According to $[12,14]$,

$$
\Sigma\left[u \mid u_{\infty}\right] \leq \frac{1}{2} \int_{\mathbb{R}^{N}} u\left|\nabla \sigma^{\prime}(u)-\nabla \sigma^{\prime}\left(u_{\infty}\right)\right|^{2} d y
$$

by the generalized Sobolev inequality, thus giving

$$
\frac{d \Sigma}{d s} \leq-2 \Sigma\left[u(\cdot, s) \mid u_{\infty}(\cdot, s)\right]+\frac{m}{m-1} \int_{\mathbb{R}^{N}}\left[u^{m-1}-u_{\infty}^{m-1}\right] F d y .
$$


To control the second term of the right hand side of the above inequality, we use Corollary 2 , and we obtain

$$
\frac{d \Sigma}{d s} \leq-2 \Sigma+\Sigma^{\frac{m-1}{m}}\|F(\cdot, s)\|_{m}
$$

This can be rewritten as

$$
\frac{d}{d s}\left[g^{\frac{1}{m}}(s)\right] \leq \frac{1}{m} e^{\frac{2}{m} s}\|F(\cdot, s)\|_{m},
$$

for $g(s):=e^{2 s} \Sigma(s)$, which by integration gives

$$
\Sigma(s) \leq e^{-2 s}\left[\Sigma^{\frac{1}{m}}(0)+\frac{1}{m} \int_{0}^{s} e^{\frac{2}{m} \tau}\|F(\cdot, \tau)\|_{m} d \tau\right]^{m} .
$$

Then the result follows using the Csiszár-Kullback type inequality stated in Proposition 2.

Acknowledgments. This work has been partially supported by the KBN grant 2 P03A 002 24, the EU financed network HPRN-CT-2002-00282 and by the European Commission Marie Curie Host Fellowship for the Transfer of Knowledge "Harmonic Analysis, Nonlinear Analysis and Probability" MTKD-CT-2004-013389.

\section{References}

[1] A. Arnold, P. Markowich, G. Toscani and A. Unterreiter, On convex Sobolev inequalities and the rate of convergence to equilibrium for Fokker-Planck type equations, Comm. Partial Differential Equations 26 (2001), 43-100.

[2] A. Arnold, P. Markowich, G. Toscani and A. Unterreiter, On generalized Csiszár-Kullback inequalities, Monatsh. Math. 131 (2000), 235-253.

[3] D. Bakry and M. Émery, Diffusions hypercontractives, in: Séminaire de probabilités, XIX, 1983/84, Lecture Notes in Math. 1123, Springer, Berlin, 1985, 177-206.

[4] N. Ben Abdallah and J. Dolbeault, Relative entropies for kinetic equations in bounded domains, Arch. Ration. Mech. Anal. 168 (2003), 253-298.

[5] R. Benguria, PhD Thesis, Princeton University (1979).

[6] R. Benguria, H. Brézis and E. H. Lieb, The Thomas-Fermi-von Weizsäcker theory of atoms and molecules, Comm. Math. Phys. 79 (1981), 167-180.

[7] P. Biler, J. Dolbeault and M. J. Esteban, Intermediate asymptotics in $L^{1}$ for general nonlinear diffusion equations, Appl. Math. Letters 15 (2001), 101-107.

[8] P. Biler, M. Guedda and G. Karch, Asymptotic properties of solutions of the viscous Hamilton-Jacobi equations, J. Evol. Equ. 4 (2004), 75-97.

[9] M. J. Cáceres, J. A. Carrillo, and J. Dolbeault, Nonlinear stability in $L^{p}$ for a confined system of charged particles, SIAM J. Math. Anal. 34 (2002), 478-494.

[10] E. A. Carlen, Superadditivity of Fisher's information and logarithmic Sobolev inequalities, J. Funct. Anal. 101 (1991), 194-211.

[11] J. A. Carrillo, A. Jüngel, P. A. Markowich and G. Toscani, A. Unterrreiter, Entropy dissipation methods for degenerate parabolic problems and generalized Sobolev inequalities, Monatsh. Math. 133 (2001), 1-82.

[12] J. A. Carrillo and G. Toscani, Asymptotic $L^{1}$-decay of solutions of the porous medium equation to self-similarity, Indiana Univ. Math. J. 49 (2000), 113-142. 
[13] I. Csiszár, Information-type measures of difference of probability distributions and indirect observations, Studia Sci. Math. Hungar. 2 (1967), 299-318.

[14] M. del Pino and J. Dolbeault, Best constants for Gagliardo-Nirenberg inequalities and application to nonlinear diffusions, J. Math. Pures Appl. 81 (2002), 847-875.

[15] S. Kullback, A lower bound for discrimination information in terms of variation, IEEE Trans. Information Theory 13 (1967), 126-127.

[16] W. I. Newman, A Lyapunov functional for the evolution of solutions to the porous medium equation to self-similarity, I, J. Math. Phys. 25 (1984), 3120-3123.

[17] J. Ralston, A Lyapunov functional for the evolution of solutions to the porous medium equation to self-similarity, II, J. Math. Phys. 25 (1984), 3124-3127.

[18] G. Toscani, Sur l'inégalité logarithmique de Sobolev, C. R. Acad. Sci. Paris Sér. I Math. 324 (1997), 689-694.

[19] G. Toscani, Entropy production and the rate of convergence to equilibrium for the FokkerPlanck equation, Quart. Appl. Math. 57 (1999), 521-541.

[20] J. L. Vázquez, Asymptotic behaviour for the porous medium equation posed in the whole space, J. Evol. Equ. 3 (2003), 67-118.

[21] J. L. Vázquez, Smoothing and Decay Estimates for Nonlinear Diffusion Equations of Porous Medium Type, Oxford Univ. Press, Oxford, 2006. 
